\title{
A Topological Approach to Chemical Organizations
}

\author{
Gil Benkö ${ }^{\mathrm{a}}$, Florian Centler ${ }^{\mathrm{b}}$, Peter Dittrich ${ }^{\mathrm{b}}$, \\ Christoph Flamm ${ }^{\mathrm{a}, \mathrm{d}}$, Bärbel M. R. Stadler ${ }^{\mathrm{c}}$, and \\ Peter F. Stadler a,d,e \\ ${ }^{a}$ Bioinformatics Group, Department of Computer Science, and \\ Interdisciplinary Center for Bioinformatics, University of Leipzig, \\ Härtelstraße 16-18, D-04107 Leipzig, Germany \\ \{gil,studla,xtof\}@bioinf.uni-leipzig.de \\ ${ }^{\mathrm{b}}$ Bio Systems Analysis Group, Jena Center for Bioinformatics and Department of \\ Mathematics and Computer Science, Friedrich Schiller University Jena, D-07743 \\ Jena, Germany \\ \{dittrich\}@minet.uni-jena.de \\ ${ }^{\mathrm{c}}$ Max Planck Institute for Mathematics in the Sciences \\ Inselstrasse 22, D-04103, Leipzig, Germany \\ \{stadler\}@mis.mpg . de \\ ${ }^{\mathrm{d}}$ Department of Theoretical Chemistry, University of Vienna, \\ Währingerstraße 17, A-1090 Wien, Austria \\ \{studla,xtof\}@tbi.univie.ac.at \\ e The Santa Fe Institute, 1399 Hyde Park Rd., Santa Fe NM 87501
}

\begin{abstract}
Large chemical reaction networks often exhibit distinctive features which can be interpreted as higher-level structures. Prime examples are metabolic pathways in a biochemical context. In this contribution we review mathematical approaches that exploit the stoichiometric structure, which can be seen as a particular directed hypergraph, to derive an algebraic picture of chemical organizations. We then give an alternative interpretation in terms of set-valued set-functions the encapsulate the production rules of the individual reactions. From the mathematical point of view, these functions define generalized topological spaces on the set of chemical species. We show that organization-theoretic concepts also appear in a natural way in the topological language. This abstract representation in turn suggest to explore the chemical meaning of well-established topological concepts. As an example, we consider connectedness in some details.
\end{abstract}

Key words: Chemical reaction networks, hypergraphs, stoichiometric matrix, flux analysis, generalized topology, closure functions, organization theory, connectedness. 


\section{Introduction}

A distinguishing feature of chemistry is that the changes of molecules upon interaction are not limited to quantitative physical properties such as free energy, density, or concentrations, since molecular interactions not only produce more of what is already there. Rather, novel molecules can be generated. This is the principal difficulty for any theoretical treatment. Dynamical systems models of chemistry start from a fixed set a molecular species and trace the time-dependence of their concentrations, in the same manner as population dynamics describes frequencies of genes or organisms. In this picture, molecules, genes, and biological species are reduced to mere indices of the concentration variables, while their properties are implicitly described by rate constants that must be determined outside these theories. On the other hand, one (or maybe the) interesting question in chemisty is which (novel) molecules arise in a given situation.

Walter Fontana and Leo Buss $[23,24]$ introduced the term constructive $d y$ namical system for such a setting in which (structural) innovation is a defining property of dynamics. In the context of a theory for biological organization they identified and discussed in detail the lack of a theory for constructive dynamical systems. Their work emphasizes an algebraic point of view, in which the interactions, i.e., in the chemical context, the reactions, are viewed as algebraic operations. Walter Fontana's AlChemy [22,24], for example represents molecules as $\lambda$-calculus expressions and reactions are defined by the operations of "application" of one $\lambda$-term to its reaction partner. The result is a new $\lambda$-term. It is worth noting in this context that chemical reactions can in turn be regarded as a model of computation, a possibility that is realized e.g. in the Chemical Abstract Machine [7]. A wide variety of different computational paradigms has been used in artifical chemistry models from strings and matrices to Turing machines and graphs $[2,3,6,18,53,63,70,71]$. We refer to [17] for a review.

Large-scale chemical reaction networks arise in very different situations, from the metabolic networks of living cells [21] to the chemistry of planetary atmospheres [75] and combinatorial chemistry, see e.g. [43]. Furthermore, artificial chemistry models at various levels of realism can be used to generate very large reaction networks, with the particular aim to determine generic properties [5]. This begs the question to analyze large-scale structures in such networks.

The first approaches towards a systematic understanding of chemical reaction networks were based on an analysis of stoichiometry in mass action reaction systems $[11,20,40,48,49,60]$. Results such a the deficiency-zero theorem provide a link between network structure and reaction kinetics [19,33, 46,47]. Metabolic flux analysis $[11,21,41,57]$ developed analogous concepts, such as 
"flux modes" that describe the dominant reaction pathways within the network. From the mathematical point of view the problem is to find the extremal rays of a cone that is determined by the stoichiometric matrix $\mathbf{S}$, see below for details. The extremal flux vectors are closely related to the (directed) cycles of the network graph [30]. The theory of Petri nets [55] and oriented matroids [54] is mathematically closely related.

Particular classes of chemical reaction networks, namely those in which reactions are catalyzed by other members of the network, are of particular interest in context of origin-of-life models $[44,50]$. The emergence of autocatalytic sets is investigated in terms of a closure function that identifies self-sustaining set where every reaction is catalyzed by a molecule in the set [45].

Based on Fontana's ideas, Peter Dittrich and Pietro Speroni di Fenizio recently presented a rigorous mathematical theory of Chemical Organizations based on notions of self-maintenance and closure [16]. From a mathematical point of view, their work emphasizes lattice theoretic structures.

Concepts from point set topological appear to have been rarely applied to chemical reaction networks. Some of us argued, motivated by applications of topological concepts in evolutionary biology $[13,25,26,66,67,73]$, that a chemical system can be meaningfully described by a generalized closure function that determines what can be constructed from a given set of molecular species [64]. Such a closure function defines in a natural way a topological structure on a chemical reaction system. In this contribution we discuss the connections between the Chemical Organizations and generalized topologies in some detail.

\section{Chemical Reactions}

Let $X$ be a (finite or infinite) set of chemical species (molecular types). A chemical reaction on $X$ is a pair of formal linear combinations of elements of $X$ :

$$
\rho: \sum_{x} \bar{\alpha}_{x, \rho} x \rightarrow \sum_{x} \alpha_{x, \rho} x, \quad \text { with } \quad \alpha_{x, \rho}, \bar{\alpha}_{x, \rho} \geq 0 .
$$

For each reaction $\rho$ we define its domain (the set of educts) and its image (the set of products)

$$
\begin{aligned}
\operatorname{dom} \rho & =\left\{x \in X \mid \bar{\alpha}_{x, \rho}>0\right\} \\
\operatorname{img} \rho & =\left\{x \in X \mid \alpha_{x, \rho}>0\right\}
\end{aligned}
$$

We say that $\rho$ is catalyzed if both $\bar{\alpha}_{x, \rho}>0$ and $\alpha_{x, \rho}>0$ for some $x \in X$. In this case, $x$ is a catalyst of $\rho$. The set of catalysts of $\rho$ is thus $\operatorname{dom} \rho \cap \operatorname{img} \rho$. Chemical reactions are often modeled as pairs of multisets (e.g. in [16]), corresponding 


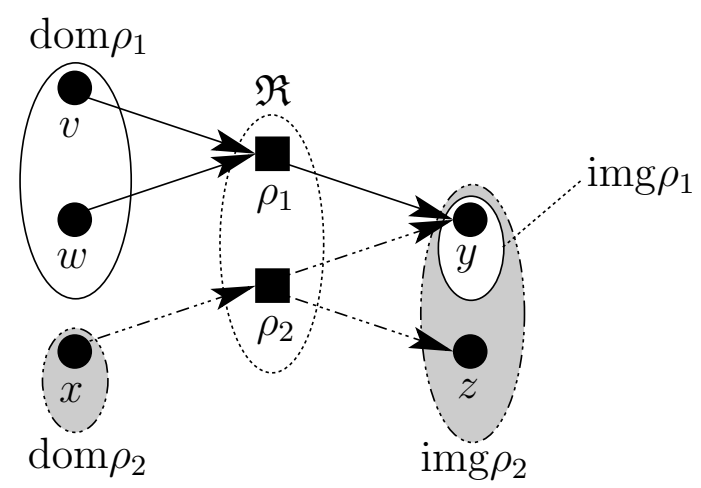

Fig. 1. The chemical universe $(X, \mathcal{R})$ for the chemical reaction network coposed of the two reactions $\rho_{1}: v+w \rightarrow y$ and $\rho_{2}: x \rightarrow y+z$ is the dupel of the set of species $X=\{v, w, x, y, z\}$ and the set of chemical reactions $\mathcal{R}=\left\{\rho_{1}, \rho_{2}\right\}$.

to integer stoichiometric constants. There does not seem to be a technical or computational advantage associated with this restriction, however, so that we allow arbitrary non-negative values of $\alpha_{x, \rho}$ and $\bar{\alpha}_{x, \rho}$.

A chemical universe is a pair $(X, \mathfrak{R})$, where $\mathfrak{R}$ is a set of chemical reactions on $X$. It can be represented by an arc-weighted bipartite directed graph $\Gamma(X, \mathfrak{R})$ with vertex set $X \cup \Re$, arcs $x \rightarrow \rho$ with weight $\bar{\alpha}_{x, \rho}$ if $\bar{\alpha}_{x, \rho}>0$, and $\operatorname{arcs} \rho \rightarrow x$ with weight $\alpha_{x, \rho}>0$ if $\alpha_{x, \rho}>0$.

We will regard all reactions as irreversible. Reversible chemical reactions are thus represented as two different reactions $\rho$ and $\bar{\rho}$ in $\mathfrak{R}$ satisfying

$$
\bar{\alpha}_{x, \bar{\rho}}=\alpha_{x, \rho} \quad \text { and } \quad \alpha_{x, \bar{\rho}}=\bar{\alpha}_{x, \rho}
$$

This framework is general enough to treat open multi-phase systems: Chemical species in different phases or compartments are treated as different molecular types. Transport between phases is represented by reactions of the form

$$
\tau: x_{i} \rightarrow x_{j}
$$

A special type of transport reactions is influx into the system and outflux from the system, represented by

$$
\begin{aligned}
& \varphi_{x}: \varnothing \rightarrow x \\
& \psi_{x}: x \rightarrow \varnothing
\end{aligned}
$$

Note that we do not assume any internal structure of the chemical species $x$. Thus, our formalism does not incorporate conservation of mass or atom types. This is convenient, since it allows us to deal e.g. with replicator systems [42] of the form

$$
I_{k}+I_{l} \rightarrow 2 I_{k}+I_{l}
$$




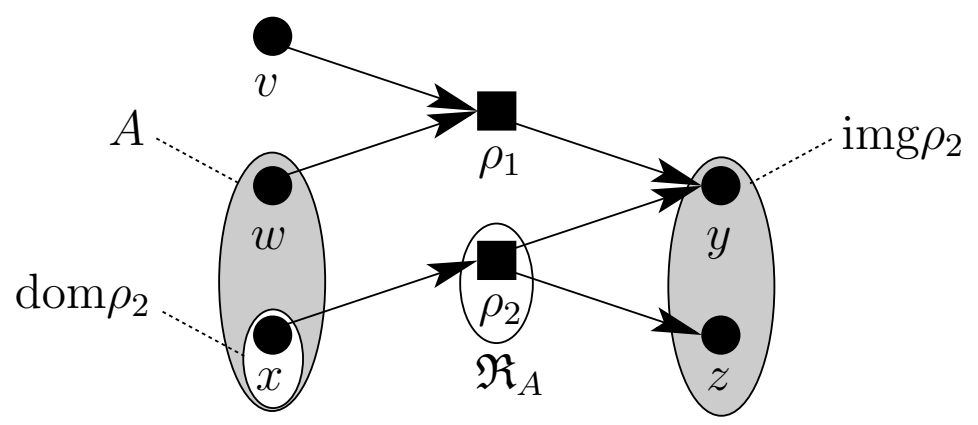

Fig. 2. If only a subset $A$ of all chemical species $X$ is present in the reaction mixture, than only those reactions can take place whose educts $\operatorname{dom} \rho_{i}$ are element of $A$. This is true for $\rho_{2}$ only in the shown reaction network.

To be chemically plausible, such a system must consume building material for the second copy of the template $I_{k}$ in produce low-energy waste. One commonly assumes in this context that the building material is "buffered", i.e., supplied externally at a constant concentration, and that the waste is removed and does not influence with the replication process. For our purposes, (6) is therefore a perfectly valid "chemical" reaction. The formalism developed here is therefore also applicable to artificial chemistry models.

Let us now consider an arbitrary subset $A \subseteq X$. Clearly, a chemical reaction $\rho$ can take place in a reaction mixture composed of the molecules in $A$ if and only if $\operatorname{dom} \rho \subseteq A$. The collection of all possible reactions in the universe $(X, \mathfrak{R})$ that can start from $A$ is thus given by

$$
\mathfrak{R}_{A}=\{\rho \in \mathfrak{R} \mid \operatorname{dom} \rho \subseteq A\}
$$

\section{Stoichiometric Matrix and Flux Vectors}

The first formal approach to analyzing the structure of complex reaction networks was probably Clarke's Stoichiometrix Network Analysis [11]. As in related lated developments such as Metabolic Flux Analyis (see e.g. the book [21] and $[28,57-59]$ recent developments in this area), its mathematical starting point is the stoichiometric matrix $\mathbf{S}$.

If $X$ and $\mathfrak{R}$ are finite, it makes sense to define the stoichiometric matrix $\mathbf{S}$ of $(X, \Re)$ with entries

$$
s_{x, \rho}=\alpha_{x, \rho}-\bar{\alpha}_{x, \rho} .
$$

Even if $X$ and $\mathfrak{R}$ are infinite it is still meaningful to introduce a stoichiometric matrix $\mathbf{S}_{A}$ on $A$ in the same way for all $x \in A$ and $\rho \in \mathfrak{R}_{A}$ provided these two sets at least are finite. 


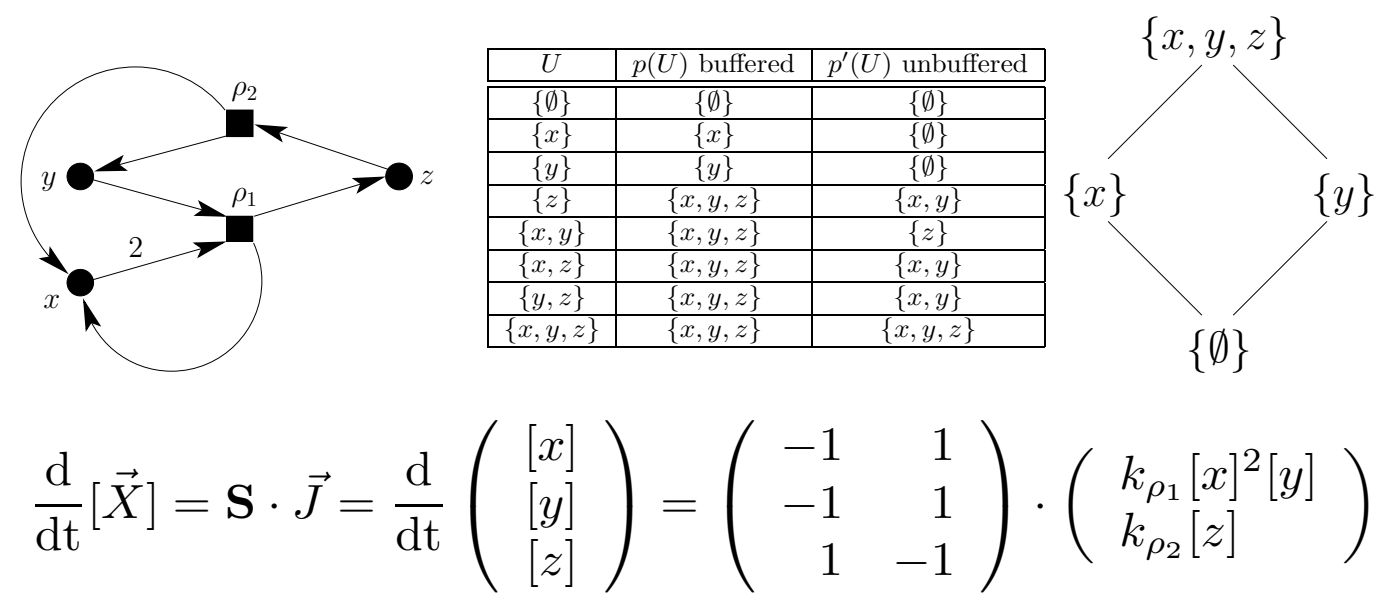

Fig. 3. Reaction network composed of the two reactions $\rho_{1}: 2 x+y \rightarrow z+x$ and $\rho_{2}: z \rightarrow x+y$ (left chart). The lattic of organisations for the reaction network (right chart). Note, that catalysts $\left(x\right.$ in reaction $\rho_{1}$ ) vanish in the stoichiometric matrix $\mathbf{S}$ since the entry for a particular chemical species $i$ is the difference $\bar{\alpha}_{i}-\alpha_{i}$ of the stoichiometric coefficients of the product and the educts side of the reaction equation. The table in the middle displays two closure functions $p$ and $p /$ applied to different sets of chemical species $U$.

We say that $x$ is produced by reaction $\rho$ if $s_{x, \rho}>0$ and that it is consummed by $\rho$ is $s_{x, \rho}<0$.

For our purposes it will also be convenient to work with a modified stoichiometric matrix $\hat{\mathbf{S}}_{A}$ of $\left(X, \Re_{A}\right)$ that in addition includes outflux reactions for all $x \in X$. The entries of $\hat{\mathbf{S}}_{A}$ are $s_{x, \rho}$ for all $x \in X$ and all $\rho \in \mathfrak{R}_{A}$ except outflux reactions and additional entries $s_{x, \psi_{x}}=-1$ for the outflux reactions of all $x \in X$.

A flux vector $J$ is a non-negative vector indexed by the reactions $\rho \in \mathfrak{R}$. In passing we note that several authors allow negative fluxes in the case of reversible reactions $[41,57]$; instead, we represent reversible reactions as a pair $\rho, \bar{\rho}$. The entries $\mathbf{S} J$ are the production or consumption rates of chemical species, i.e., the kinetics of a chemical reaction can be always tbe written in the form

$$
\frac{\mathrm{d}}{\mathrm{d} t}[x]=(\mathbf{S} J)_{x}
$$

A flux vector $J$ is called stationary if $\mathbf{S} J=\vec{o}$. Obviously, stationary flux vectors correspond to fixed points of the kinetic equations (9).

We immediately observe the following relationships between $\mathbf{S}_{A}$ and $\hat{\mathbf{S}}_{A}$ : A flux vector $J$ can be extended to a stationary flux vector w.r.t. $\hat{\mathbf{S}}_{A}$ if and only if $\left(\mathbf{S}_{A} J\right)_{x} \geq 0$ for all $x \in A$. 

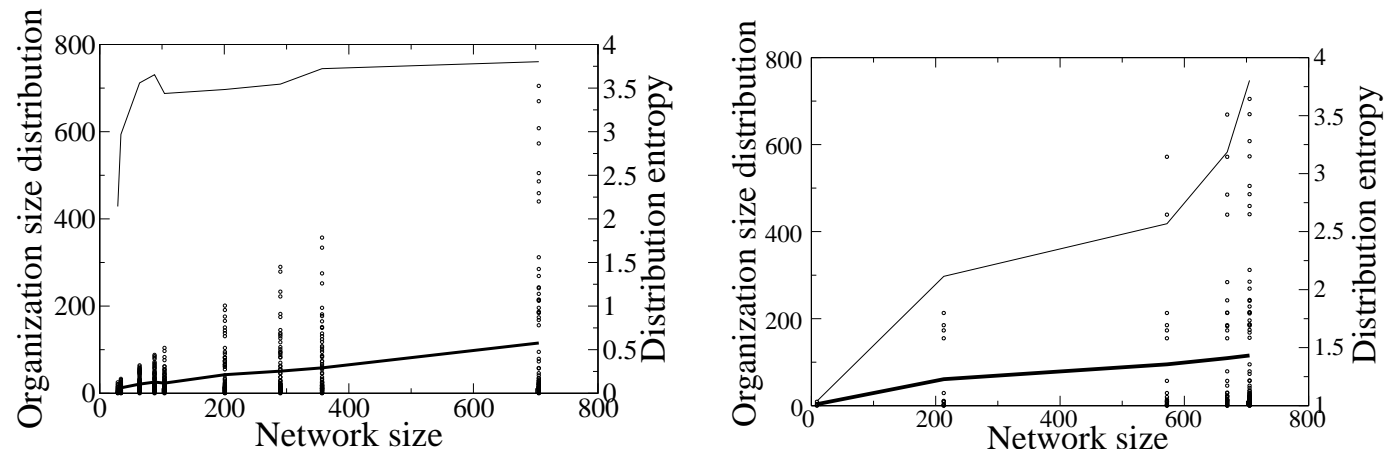

Fig. 4. Change of the set of organizations with a growing network, by increasing the size of molecules allowed (left chart), or the number of starting molecules (right chart). For each network size, the sizes of all organizations form a distribution (o) which is shown with its mean (connected by the bold black line) and its entropy (connected by a black line).

\section{Chemical Organizations}

In [16] a formal Theory of Chemical Organizations is outlined based on the following definitions:

(1) A subset $A \subset X$ is "closed" 1 if for all reaction $\rho$ with $\operatorname{dom} \rho \subseteq A$ we have $\operatorname{img} \rho \subseteq A$.

(2) The "closure" $C(A)$ of a set $A$ is the smallest "closed" set containing $A$. A similar closure function plays a crucial role in the theory of "catalytic reaction systems" by Mike Steel and Wim Hordijk [44].

(3) A set $A$ is semi-self-maintaining if every species $x$ that is "used-up" (i.e., there is a reaction $\theta \in \mathfrak{R}_{A}$ such that $s_{x, \theta}<0$ ) is also produced (i.e., there is a reaction $\rho \in \mathfrak{R}_{A}$ such that $\left.s_{x, \rho}>0\right)$.

(4) A set $A$ is called a semi-organization if it is "closed" and semi-selfmaintaining.

(5) A set $A$ is called self-maintaining if there exists a flux vector with the following properties: (1) $J_{\rho}>0$ for all $\rho \in \mathfrak{R}_{A}$, (2) $J_{\rho}=0$ for all $\rho \notin \mathfrak{R}_{A}$, (3) $(\mathbf{S} J)_{x} \geq 0$ for all $x \in A$.

(6) A set $A$ is called an organization if it is "closed" and self-maintaining.

The exposition [16] is concerned mostly with lattice-theoretic considerations, such as conditions under which the set of all organizations forms of $(X, \mathfrak{R})$ forms a lattice. In this contribution we shall explore a different mathematical angle.

1 We use quotes here to distinguish the definition of a closed set in the sense of the theory of Chemical Organizations from the topological notion of a closed set". As we shall see, this is related but not quite the same. 
We generated two series of networks of increasing size using the ToyChem Model. There, a chemical reaction network is generated by starting with a list of seed molecules and predicting the outcomes of the reactions between them, and repeating this process iteratively on the resulting molecules. For the first series, the complexity of the chemistry increased at each step by adding new chemical species to the starting molecules, as opposed to the second series, where the molecular size threshold above which reactions were neglected was increased. Thus in the former case new chemical species are introduced without allowing new reactions between old chemical species at each step, whereas in the latter case new reactions are allowed at each step. We expect that an organization is preserved when only new chemical species are introduced. Indeed, this is nicely illustrated by the first resulting series of sets of organizations, as seen in Fig. 4 (right chart). In contrast to Fig. 4 (left chart), the lattice of organizations of a smaller subnetwork is a sublattice of the lattice of organizations of the a bigger network. Organizat ion theory thus lets us study the modularity of a reaction network. Whereas some networks lead to the complex lattice shown in Fig. 4, the purine synthesis network of [4] leads only to a set of organizations composed of the empty set and the complete network. This seems to indicate that the purine synthesis there is not modular.

\section{Set-Valued Set-Functions}

In [64] we considered the set of molecular types that can be produced by chemical reactions from subsets $A \in \mathcal{P}(X)^{2}$ as a function of the set $A$. Using the notation introduced in the previous section, this function can be written as

$$
p^{\prime}(A)=\bigcup_{\rho \in \Re_{A}} \operatorname{img} \rho
$$

A slightly more restrictive definition takes into account that a molecular species $x$ may be effectively destroyed in a reaction $\rho$ but still appear in on the r.h.s. of the chemical equation. This is the case if $0<\alpha_{x, \rho}<\bar{\alpha}_{x, \rho}$. Thus we set

$$
\tilde{p}^{\prime}(A)=\bigcup_{\rho \in \Re_{A}}\left\{x \in X \mid s_{x, \rho}>0\right\}
$$

These functions, however, have the sometimes undesirable property of excluding inert species that only act as catalysts or that are not touched my any reaction, i.e., the set

$$
k(A)=\left\{x \in A \mid s_{x, \rho}=0 \forall \rho \in \mathfrak{R}_{A}\right\}
$$

$\overline{2 \mathcal{P}(X)}$ is the power set, i.e., the set of all subsets of $X$. 


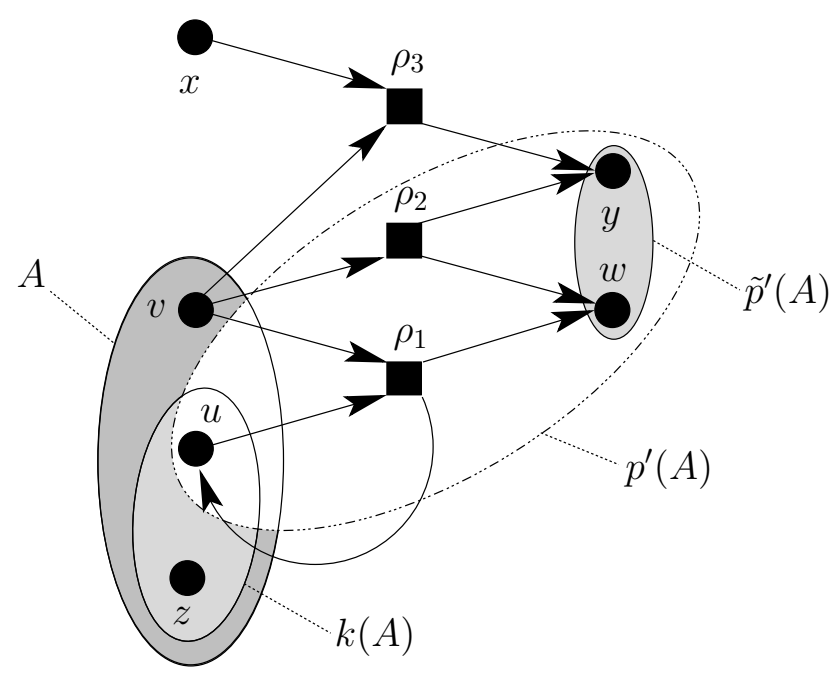

Fig. 5. Illustration of the set-valued set-functions $k, p^{\prime}, \tilde{p}^{\prime}$ for the subset $A=\{u, v, z\}$ with $u$ being a catalyst and $z$ an inert species.

In many situation it will be more natural to consider

$$
p(A)=p^{\prime}(A) \cup k(A) \text { and } \tilde{p}(A)=\tilde{p}^{\prime}(A) \cup k(A)
$$

Formally, $p, p^{\prime}, \tilde{p}$, and $\tilde{p}^{\prime}$ are functions $\mathcal{P}(X) \rightarrow \mathcal{P}(X)$, so called set-valued set functions. In a sense, these functions describe the structure of the chemical network on an "aggregate" level by the compound outcome of reaction mixtures.

Comparing these definitions and the definition of "closed" and semi-selfmaintaining sets we find:

Lemma $1 A$ set $A$ is "closed" if and only if $p^{\prime}(A) \subseteq A$. $A$ set $A$ semi-selfmaintaining if and only if $A \subseteq \tilde{p}(A)$.

Proof: Recall, that $A$ is "closed" if and only if $\operatorname{img} \rho \subseteq A$ whenever $\rho \in \mathfrak{R}_{A}$. Now suppose $A$ is semi-self-maintaining. If $x \in A$ is used up is some reaction then it must be produced in some reaction, i.e., $x \in \tilde{p}^{\prime}(A)$. If $x$ is not used up by any reaction then $s_{x, \rho} \geq 0$ for all $\rho \in \mathfrak{R}_{A}$, i.e., either $x \in k(A)$ or there is a reaction $\tau$ with $s_{x, \tau}>0$, in which case $x \in \tilde{p}^{\prime}(A)$. It follows that in each case $x \in \tilde{p}(A)$. Conversely, suppose $x \in \tilde{p}(A)$. Then $\in \tilde{p}^{\prime}(A)$, i.e., $x$ is produced by some reaction, or $x \in k(A)$, i.e., $x$ is not consumed by any reaction. If follows that $A$ is semi-self-maintaining.

Since the definitions of $p^{\prime}(A)$ and $\tilde{p}^{\prime}(A)$ differ only in their treatment of some species $x \in A$ we observe $p^{\prime}(A) \subseteq A$ if and only if $\tilde{p}^{\prime}(A) \subseteq A$. Furthermore, we have always $k(A) \subseteq A$. Thus we obtain 
Theorem 1 A subset $A \subseteq X$ is a semi-organization of $(X, \Re)$ if and only if $\tilde{p}(A)=A$.

Lemma 1 suggests to consider other set-valued set-functions that might be used to characterize self-maintenance. We shall see that this approach in a rather natural way leads to a family of such functions.

We say $x \in A$ is maintainable in $A$ if for each $\rho \in \mathfrak{R}_{A}$ with $x \in \operatorname{dom} \rho$ there is a flux vector $J$ such that (1) $J_{\rho}>0$ and (2) $\hat{\mathbf{S}}_{A} J=\vec{o}$. In other words, $x$ is maintainable in $A$ if no sustainable reaction in $\rho \in \mathfrak{R}_{A}$ invitable leads to the depletion of $x$. In particular, $x$ in maintainable in $A$ if it is not required for any reaction of $A$ at all. Let $m(A)$ denote the set of maintainable species in $A$.

Lemma $2 A$ set $A$ is self-maintaining if and only if each $x \in A$ is maintainable in $A$, i.e., if $m(A)=A$.

Proof: By taking a suitable positive (convex) combination we see that there is a flux vector $J^{x}$ satisfying $\hat{\mathbf{S}}_{A} J^{x}=\vec{o}$ and $J_{\rho}^{x}>0$ for all $\rho \in \mathfrak{R}_{A}$ with $x \in \operatorname{dom} \rho$. Taking again a a suitable positive convex combination we can construct a stationary flux vector $J^{*}$ (w.r.t. $\hat{\mathbf{S}}_{A}$ such that $J_{\rho}^{*}>0$ for all $\rho \in \mathfrak{R}_{m}(A)$. This $m(A)=A$ is equivalent to the existence of a flux vector $J$ for which (1) $J_{\rho}>0$ for all $\rho$ in $\mathfrak{R}_{A}$ and (2) $(\mathbf{S} J)_{x} \geq 0$ for all $x \in A$, i.e., to the fact that $A$ is self-maintaining.

Lemma 3 For all $A \subseteq X$ holds $m(A) \subseteq \tilde{p}(A)$.

Proof: If $x \in m(A)$ than there is a stationary flux vector satisfying $J_{\rho}>0$ for all $\rho \in \mathfrak{R}_{x}:=\left\{\rho \in \mathfrak{R}_{A} \mid x \in \operatorname{dom}_{\rho}\right\}$. If $\mathfrak{R}_{x}=\emptyset$ then $x \in k(A)$. If this is not the case than either $s_{x, \rho}=0$ for all $\rho \in \mathfrak{R}_{x}$ (in which case $x \in k(A)$ again), or there is at least one reaction $\rho$ with $s_{x, \rho} \neq 0$. Since $J$ is stationary with $J_{\rho}>0$ for all $\rho$, in particular those with $s_{x, \rho}<0$, it follows that there is at least one reaction $\tau \in \mathfrak{R}_{A}$ with $s_{x, \tau}>0$ (otherwise $J$ could not be stationary). In this last case $x \in \tilde{p}^{\prime}(A)$.

The construction of $m(A)$ is somewhat unsatisfactory because it does not distinguish between those species which can be sustainably produced from the set $A$ and it does not consider those by-products that are produced outside of $A$. Such a modified concept would be of particular interest e.g. in the context of metabolic networks.

Let us say that $x \in X$ is stationarily produced from $A$ if there is a stationary flux vector $J$ (w.r.t. $\hat{\mathbf{S}}_{A}$ ) such that $J_{\psi_{x}}>0$, i.e., if the stationary flux produces an excess of $x$ that we have to remove using the outflux reaction $\psi_{x}$ in order to maintain balance. Note that we do not require each reaction with $x \in \operatorname{dom} \rho$ to have non-zero flux here. In fact, e.g. in the context of metabolic networks 
it makes sense to assume that we can "switch off" certain reaction channels.

Now define $s(A)$ as the set of all species that are stationarily produced from $A$. It is easy to show that there is a stationary flux vector that simultaneously produces all $x \in s(A)$ : For each $x \in s(A)$, find a flux vector $J_{x}$ that produces $x$ and take a strictly positive convex combination $J^{*}$ of these vectors. Clearly $J^{*}$ is again a stationary flux vector on $A$ and by construction $J_{\psi_{x}}>0$ for all $x \in s(A)$. The set of all such stationary flux vectors froms a cone $\mathcal{K}_{s}$.

By construction, $x \in s(A)$ implies that there is at least one reaction $\rho \in \mathfrak{R}_{A}$ that produces $x$, i.e., $s_{\rho, x}>0$ for some $\rho \in \mathfrak{R}_{A}$. Consequently, $s(A) \subseteq \tilde{p}^{\prime}(A)$.

An even stronger requirement on the flux vectors is to assume that there is inevitably a loss in all species that are produced. Formally, this means

$$
J_{\psi_{x}}>0 \text { for all } x \in \bigcup_{\rho: J_{\rho}>0} \operatorname{img} \rho
$$

Let $s^{*}(A)$ be the set of all $x \in X$ such that there is a stationary flux vector $J$ on $A$ satisfying conditions (14). By the same argument as above there is a stationary flux vector $J^{*}$ satisfying (14) and $J_{\psi_{x}}>0$ for all $x \in s^{*}(A)$ and an associated cone $\mathcal{K}_{s^{*}}$. By construction, $\mathcal{K}_{s^{*}} \subseteq \mathcal{K}_{s}$. This implies $s^{*}(A) \subseteq s(A)$.

It is sometimes convenient to consider only those species $x$ that are stationarily produced within a set $A$, i.e., $x \in A \cap s(A)$. Clearly, any such species can also be maintained within $A$. Thus we have $A \cap s(A) \subseteq m(A)$.

Let us summarize the mutual relationships of the set functions defined so far:

Theorem 2 For all $A \subseteq X$ we have the following system of inclusions:

$$
\begin{aligned}
& A \cap s(A) \subseteq m(A) \subseteq \tilde{p}(A) \subseteq p(A) \\
& s^{*}(A) \subseteq s(A) \subseteq \tilde{p}^{\prime}(A) \subseteq p^{\prime}(A)
\end{aligned}
$$

\section{Isotonic and Non-Isotonic Functions}

One of the most basic (and desirable) properties of set-valued set-functions is isotony:

A function $u: \mathcal{P}(X) \rightarrow \mathcal{P}(X)$ is isotonic if $A \subseteq B$ implies $u(A) \subseteq u(B)$.

Theorem $3 p^{\prime}, \tilde{p}^{\prime}, s$ and $s^{*}$ are isotonic functions. 


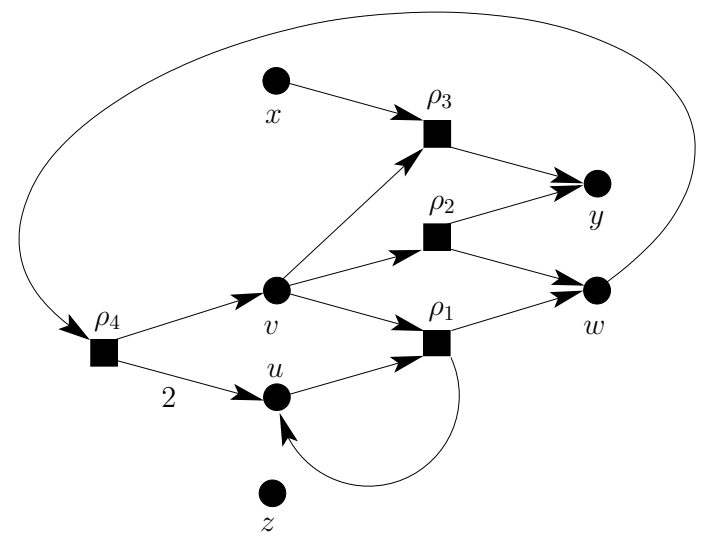

Fig. 6. Illustration of the set-valued set-functions $m, s$ and $s^{*}$ for the set $A=\{u, v, w, x, y, z\}$. The reaction network is essentially the one from Fig 5 with the additional reaction $\rho_{4}$. The species $x$ and $v$ are not maintainable. The set of selfmaintaining chemical species is $m(A)=\{u, w, y, z\}$. If outflow is applied to the produced species in the set $A$ the set of maintainable species reduces to the set of stationary produced species $s(A)=\{u\}$. If outflow is applied to all species in $A$ the set of stationary produced species reduces to $s^{*}$ which in this case is the empty set.

Proof: It is easy to see that $p^{\prime}$ is isotonic. If $x \in p^{\prime}(A)$ then there here is a reaction $\rho$ with $\operatorname{dom} \rho \subseteq A$ and $x \in \operatorname{img} \rho$. It follows that $\operatorname{dom} \rho \subseteq B$ and hence $x \in p(B)$. An analogous argument works for $\tilde{p}^{\prime}$.

In order to show the analogous results for $s$ and $s^{*}$ we need to make sure that enlarging the subset $A$ does not interfer with the stationary flux vectors. The following diagram shows the structure of the modified stoichiometric matrix $\hat{\mathbf{S}}_{B}$. The diagonal block at the right hand side denotes the outflux reactions $\psi_{x}$ for each $x \in X$ with entry -1 .

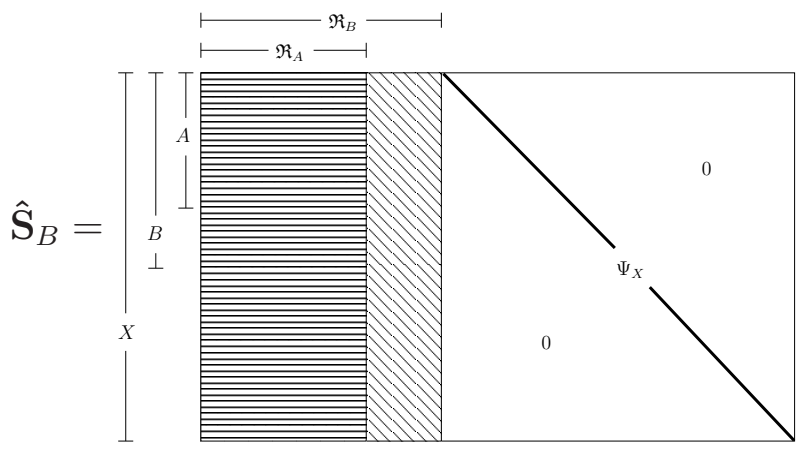

We see that $\hat{\mathbf{S}}_{A}$ is a submatrix of $\hat{\mathbf{S}}_{B}$ with a very simple structure: the columns representing the non-outflux reactions in $\mathfrak{R}_{B} \backslash \mathfrak{R}_{A}$ are missing, otherwise the two matrices coincide. A solution of $\hat{\mathbf{S}}_{A} J=0$ thus has a corresponding solution $\hat{\mathbf{S}}_{B} J^{\prime}=0$, where $J^{\prime}$ is obtained from $J$ by adding 0 -entries to the $\mathfrak{R}_{B} \backslash \mathfrak{R}_{A^{-}}$ coordinates; otherwise $J$ and $J^{\prime}$ are identical. Clearly, we have $J_{\rho}>0$ only for reactions with $\operatorname{dom} \rho \in A \subset B$ (except for outflux reactions), this $J^{\prime}$ is a 
stationary flux vector on $B$. Since $J$ and $J^{\prime}$ coincide on the outflux reactions, we have conclude that $x \in s(A)$ implies $x \in S(B)$.

Furthermore, if $J$ satiesfies (14), so does $J^{\prime}$, and hence $x \in s^{*}(A)$ implies $x \in s^{*}(B)$.

In contrast, however, $p, \tilde{p}$, and $m$ are not isotonic in general. As a counterexample, consider the following situation: Suppose there is $x \in A$ such that $x \notin \operatorname{dom} \rho$ for all $\rho \in \mathfrak{R}_{A}$. For any flux vector $J$ satisfying the conditions in the definition of $m$ above we have $(\mathbf{S} J)_{x}=0$ and hence $x \in m(A) \subseteq \tilde{p}(A) \subseteq p(A)$. Assume furthermore, that there is a single reaction $\theta \in \mathfrak{R}_{B}$ for some $B$ satisfying $A \subset B$ that consumes $x$, i.e., $x \in \operatorname{dom} \theta$. Furthermore, suppose $x \notin \operatorname{img} \theta$. Clearly, $x \notin p(B)$ and hence also not in $\tilde{p}(B)$, which in turn implies $x \notin m(B)$. It follows in particular that $p(A) \nsubseteq p(B), \tilde{p}(A) \nsubseteq \tilde{p}(B)$ and $m(A) \nsubseteq m(B)$.

A reaction network $(X, \mathfrak{R})$ is a flow systems if for each $x \in X$, there is an outflux reaction $\psi_{x}$. It follows immediately, that $k(A)=\emptyset$ and $\hat{\mathbf{S}}_{A}=\mathbf{S}_{A}$ for all $A \in \mathcal{P}(X)$.

Lemma 4 Let $(X, \mathfrak{R})$ be a flow systems. Then $m: \mathcal{P}(X) \rightarrow \mathcal{P}(X)$ is isotonic.

Proof: By construction there is a flux vector $J$ satisfying $\mathbf{S}_{A} J=0, J_{\rho}>0$ for all $\rho \in \mathfrak{R}_{A}$ in particular $J_{\psi_{x}}>0$ for all $x \in m(A)$. Now consider $m(B)$. Analogously, there is a flux vector $J^{\prime}$ satisfying $\mathbf{S}_{B} J^{\prime}=0$. Now construct a flux vector $J^{*}$ such that $J_{\rho}^{*}=J+\rho+\varepsilon J_{\rho}$ for all $\rho \in \mathfrak{R}_{B}$ with the exception of the outflux reactions $\psi_{x}$ with $x \in m(A)$. The flux through the latter is adjusted such that stationarity is maintained. For small enough $\varepsilon$, this can be achieved such that $J_{\psi_{x}}^{*}>0$. It follows that $m(A) \subseteq m(B)$.

Theorem 4 Let $(X, \mathfrak{R})$ be a flow systems. Then $A$ is self-maintaining if and only if $A \subseteq s(A)$.

Proof: In a flow system, any stationary flux vector on $A$ satisfying $\left(\mathbf{S}_{A} J\right)_{x}=$ 0 for all $x \in A$ and $J_{\psi_{x}}>0$ can be modified to satisfy also $J_{\rho}>0$ for all $\rho \in \mathfrak{R}_{A}$ by reducing the outfluxes $J_{\psi_{x}}$ in return. Choosing $J_{\rho}$ small enough this can clearly be done in such a way that the that $J_{\psi_{x}}>0$. Thus, if $A \subseteq s(A)$ we have also $m(A)=A$. The converse is obvious.

We remark that this result also holds for flow systems with permanent molecules [16].

Lemma 5 Let $(X, \mathfrak{R})$ be a flow systems. Then for all $A, s(A) \cap A=m(A)$.

PRoOF: In general, a stationarily produced molecule is also maintainable in $A$, i.e., $A \cap s(A) \subseteq m(A)$. It remains to be shown that in a flow system a maintainable molecules in $A$ is stationarily produced in $A$ : In a flow system there is 
an outflux reaction $\psi_{x}$ for every molecule $x \in A$. If a molecule is maintainable, this outflux reaction can be compensated (i.e., there is a stationary flux vector $J$ with $J_{\psi_{x}}>0$ ). Therefore the molecule is also stationarily produced.

\section{Generalized Topologies}

The connection of our exposition so far and the framework of (point set) topology is the - maybe surprising - fact that some meaningful topological concepts can already be defined on a set $X$ endowed with an arbitrary setvalued set-function. This section summarizes, and in part slighly extends, results by Day [14], Hammer [29,36] and Gniłka [31] on such structures.

Let $X$ be an arbitary set and let $\mathrm{cl}: \mathcal{P}(X) \rightarrow \mathcal{P}(X)$ be an arbitrary set-valued function. We shall see that it is fruitful to interpret $\mathrm{cl}$ as a closure function on $X$; hence we call $\mathrm{cl}(A)$ is the closure of the set $A$. The dual of the closure function is the interior function int $\mathcal{P}(X) \rightarrow \mathcal{P}(X)$ defined by

$$
\operatorname{int}(A)=X \backslash \operatorname{cl}(X \backslash A)
$$

Given the interior function, we obviously recover the closure as $\operatorname{cl}(A)=X \backslash$ $(\operatorname{int}(X \backslash A))$. Note that the distinction of closure and interior is completely arbitrary in the absence of additional conditions.

The most immediate connection between chemical organizations and topological concepts is provided by the "closure" function $C(A)$ defined in [16], and denoted $G_{C L}($.$) in this paper.$

On the other hand, we might as well use any of the set-valued set-functions defined in the previous sections to capture the topologial structure of a chemical reaction network. We argue that the most natural choice is the closure function

$$
\mathrm{cl}(A)=p(A) \cup A=\tilde{p}(A) \cup A=p^{\prime}(A) \cup A=\tilde{p}^{\prime}(A) \cup A .
$$

Indeed, $\mathrm{cl}(A)$ describes the state of the chemical system after the products obtainable from $A$ have been produced but before the original material has been used up completely. It is easy to check that $\mathrm{cl}$ is isotonic and enlarging.

The function cl also has an easy relation with the "closure" $C(A)$ : By construction $A \subseteq C(A)$. Since $C(A)$ is closed, it must contain also everything that can be generated from $A$, i.e., $p(A) \subseteq C(A)$, and hence $\mathrm{cl}(A) \subseteq C(A)$. But this implies that $C(A)=C(\mathrm{cl}(A))$ Repeating the argument, we see $\mathrm{cl}^{n}(A) \subseteq C(A)$ for all $n$. In the case of infinite sets, $n$ may be any ordinal number. It can be proved that there is in general a smallest ordinal number $\eta$ such that $\mathrm{cl}^{\eta}(A)=C(A)$. 
The closure function $\mathrm{cl}$ is implicitly used in the "generate closure" operator in [16].

In topological theory, a set $A \in \mathcal{P}(X)$ is closed if $A=\mathrm{cl}(A)$ and open if $A=\operatorname{int}(A)$. Clearly, $A$ is closed if and only if $A=C(A)$. A set $A$ is open if its complement $X \backslash A$ is closed. In contrast to "classical" topology, open and closed sets do not define the structure of our chemical universe. Nevertheless, the define of course interesting subsets.

Alternatively, we could use $i(A)=\tilde{p}(A) \cap A$ as an interior function. (Note that $i(A)$ and $\mathrm{cl}(A)$ are in general not duals!). In the resulting topological structure, semi-self-maintaining sets correspond to open sets. Repeated application of $i$ eventually (after a finite number of steps in finite sets) leads to an idempotent interior function $I(A)=i^{\eta}(A)$. In consistent reaction systems, $I(A)$ defines the largest semi-self-maintaining set contained in $A$. In this structure, semiself-maintaining sets are exactly the open sets in $(X, i)$.

Both the idempotent closure $C$ and the idempotent interior $I$ are used in explicitly in [16] as a means to generate "closed" and semi-self-maintaining sets. In fact, $\mathrm{cl}$ and $i$ are used implicitly to computationally construct $C$ and $I$, respectively.

Interestingly, any extended topology (isotonic space) can be seen as a "combination" of an expanding and a contracting isotonic function, i.e., as a closure and an interior function defining two different neighborhood spaces:

Lemma 6 Let $u: \mathcal{P}(X) \rightarrow \mathcal{P}(X)$ and $v: \mathcal{P}(X) \rightarrow \mathcal{P}(X)$ be a two isotonic functions such that $u(A) \subseteq A \subseteq v(A)$ and let $w(A)=u(A) \cup[v(A) \backslash A]$. Then $u(A)=w(A) \cap A$ and $v(A)=w(A) \cup A$.

Proof: Direct computation.

Let $\mathrm{cl}$ and int be a closure function and its dual interior function on $X$. Then the neighborhood function $\mathcal{N}: X \rightarrow \mathcal{P}(\mathcal{P}(X))$ assign to each point $x \in X$ the collections

$$
\mathcal{N}(x)=\{N \in \mathcal{P}(X) \mid x \in \operatorname{int}(N)\}
$$

of its neighborhoods. Closure and neighborhood are equivalent [14]. More precisely:

$$
x \in \mathrm{cl}(A) \Longleftrightarrow(X \backslash A) \notin \mathcal{N}(x) \quad \text { and } \quad x \in \operatorname{int}(A) \Longleftrightarrow A \in \mathcal{N}(x)
$$

Let $c^{\prime}$ and $c^{\prime \prime}$ be two generalized closure operators on $X$. We say that $c^{\prime}$ is finer than $c^{\prime \prime}, c^{\prime} \succeq c^{\prime \prime}$, or $c^{\prime \prime}$ is coarser than $c^{\prime}$ if $c^{\prime}(A) \subseteq c^{\prime \prime}(A)$ for all $A \in \mathcal{P}(X)$. Note that $c^{\prime} \succeq c^{\prime \prime}$ and $c^{\prime} \preceq c^{\prime \prime}$ implies $c^{\prime}=c^{\prime \prime}$. 


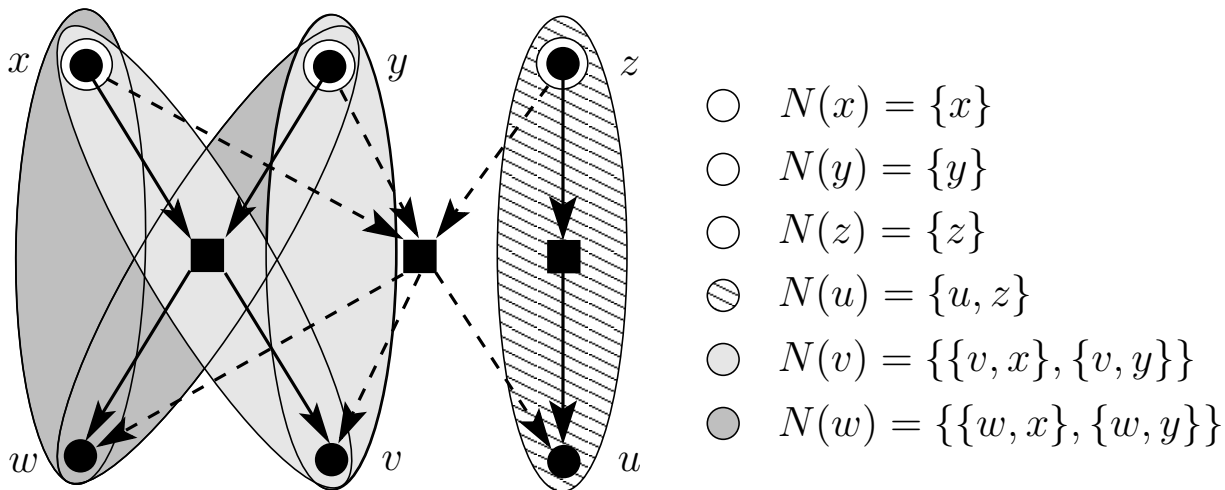

Fig. 7. Minimal neighbohoods for each of the species $i \in\{x, y, z, u, v, w\}$ in a reaction network compose of two "elementary" reactions (bold arrows) and a super-reaction (dashed arrows) which performs the same chemical transformation as the superposition of the two elementary reactions. By definition, all supersets of neighborhoods are also neighborhoods. In contrast to graphs, the minimal neighborhoods are not unique. For instance, $w$ has the two minimal neighborhoods $\{w, x\}$ and $\{w, y\}$.

A function $f:(X, \mathrm{cl}) \rightarrow(Y, \mathrm{cl})$ is

closure preserving if for all $A \in \mathcal{P}(X)$ holds $\quad f(\operatorname{cl}(A)) \subseteq \operatorname{cl}(f(A))$;

continuous $\quad$ if for all $B \in \mathcal{P}(Y)$ holds $\quad \mathrm{cl}\left(f^{-1}(B)\right) \subseteq f^{-1}(\mathrm{cl}(B))$.

It is obvious that the identity $\imath:(X, \mathrm{cl}) \rightarrow(X, \mathrm{cl}): x \mapsto x$ is both closurepreserving and continuous since $\imath(\mathrm{cl}(A))=\mathrm{cl}(A) \subseteq \mathrm{cl}(A)=\mathrm{cl}(\imath(A))$. Furthermore, the concatenation $h=g(f)$ of the closure-preserving (continuous) functions $f: X \rightarrow Y$ and $g: Y \rightarrow Z$ is again closure-preserving (continuous).

Let $(X, \mathrm{cl})$ and $(Y, \mathrm{cl})$ be two sets with arbitrary closure functions and let $f: X \rightarrow Y$. Then the following conditions (for continuity) are equivalent, see e.g. [32, Thm.3.1.]:

(i) $\mathrm{cl}\left(f^{-1}(B)\right) \subseteq f^{-1}(\mathrm{cl}(B))$ for all $B \in \mathcal{P}(Y)$.

(ii) $f^{-1}(\operatorname{int}(B)) \subseteq \operatorname{int}\left(f^{-1}(B)\right)$ for all $B \in \mathcal{P}(Y)$.

(iii) $B \in \mathcal{N}(f(x))$ implies $f^{-1}(B) \in \mathcal{N}(x)$ for all $B \in \mathcal{P}(Y)$ and all $x \in X$.

Conditions (iii) can used as a definition of continuity at as each individual point $x \in X$.

The notation of a neighborhood for an individual point can be extended naturally to sets: Let $A \in \mathcal{P}(X)$. A set $V$ is a neighborhood of $A$, in symbols $V \in \mathcal{N}(A)$ if $V \in \mathcal{N}(x)$ for all $x \in A$. Obviously, $\mathcal{N}(\{x\})=\mathcal{N}(x)$.

Lemma 7 For all $V, A \in \mathcal{P}(X)$ we have $V \in \mathcal{N}(A)$ iff and only if $A \subseteq \operatorname{int}(V)$. 
Table 1

Basic axioms for Generalized Topologies.

The properties below are meant to hold for all $A, B \in \mathcal{P}(X)$ and all $x \in X$, respectively.

\begin{tabular}{|c|c|c|c|}
\hline & closure & interior & neighborhood \\
\hline K0' & $\exists A: x \notin \mathrm{cl}(A)$ & $\exists A: x \in \operatorname{int}(A)$ & $\mathcal{N}(x) \neq \varnothing$ \\
\hline K0 & $\mathrm{cl}(\emptyset)=\emptyset$ & $\operatorname{int}(X)=X$ & $X \in \mathcal{N}(x)$ \\
\hline $\begin{array}{l}\text { K1 } \\
\text { isotonic, } \\
\text { monotone }\end{array}$ & $\begin{array}{l}A \subseteq B \Longrightarrow \operatorname{cl}(A) \subseteq \operatorname{cl}(B) \\
\mathrm{cl}(A \cap B) \subseteq \operatorname{cl}(A) \cap \operatorname{cl}(B) \\
\mathrm{cl}(A) \cup \mathrm{cl}(B) \subseteq \operatorname{cl}(A \cup B)\end{array}$ & $\begin{array}{l}A \subseteq B \Longrightarrow \operatorname{int}(A) \subseteq \operatorname{int}(B) \\
\operatorname{int}(A) \cup \operatorname{int}(B) \subseteq \operatorname{int}(A \cup B) \\
\operatorname{int}(A \cap B) \subseteq \operatorname{int}(A) \cap \operatorname{int}(B)\end{array}$ & $\begin{array}{c}N \in \mathcal{N}(x) \text { and } N \subseteq N^{\prime} \\
\Longrightarrow \\
N^{\prime} \in \mathcal{N}(x)\end{array}$ \\
\hline $\mathrm{KA}$ & $\operatorname{cl}(X)=X$ & $\operatorname{int}(\emptyset)=\emptyset$ & $\emptyset \notin \mathcal{N}(x)$ \\
\hline $\mathrm{KB}$ & $\begin{array}{l}A \cup B=X \Longrightarrow \\
\operatorname{cl}(A) \cup \operatorname{cl}(B)=X\end{array}$ & $\begin{array}{c}A \cap B=\emptyset \Longrightarrow \\
\operatorname{int}(A) \cap \operatorname{int}(B)=\emptyset\end{array}$ & $\begin{array}{c}N^{\prime}, N^{\prime \prime} \in \mathcal{N}(x) \Longrightarrow \\
N^{\prime} \cap N^{\prime \prime} \neq \emptyset\end{array}$ \\
\hline $\begin{array}{l}\mathrm{K} 2 \\
\text { expansive }\end{array}$ & $A \subseteq \mathrm{cl}(A)$ & $\operatorname{int}(A) \subseteq A$ & $N \in \mathcal{N}(x) \Longrightarrow x \in N$ \\
\hline $\begin{array}{l}\text { K3 } \\
\text { sub-linear }\end{array}$ & $\mathrm{cl}(A \cup B) \subseteq \mathrm{cl}(A) \cup \mathrm{cl}(B)$ & $\operatorname{int}(A) \cap \operatorname{int}(B) \subseteq \operatorname{int}(A \cup B)$ & $\begin{array}{c}N^{\prime}, N^{\prime \prime} \in \mathcal{N}(x) \Longrightarrow \\
N^{\prime} \cap N^{\prime \prime} \in \mathcal{N}(x)\end{array}$ \\
\hline $\begin{array}{l}\text { K4 } \\
\text { idempotent }\end{array}$ & $\operatorname{cl}(\operatorname{cl}(A))=\operatorname{cl}(A)$ & $\operatorname{int}(\operatorname{int}(A))=\operatorname{int}(A)$ & $\begin{array}{l}N \in \mathcal{N}(x) \Longleftrightarrow \\
\operatorname{int}(N) \in \mathcal{N}(x)\end{array}$ \\
\hline $\begin{array}{l}\text { K5 } \\
\text { additive }\end{array}$ & $\bigcup_{i \in I} \mathrm{cl}\left(A_{i}\right)=\mathrm{cl}\left(\bigcup_{i \in I} A_{i}\right.$ & $\bigcap_{i \in I} \operatorname{int}\left(A_{i}\right)=\operatorname{int}$ & $\begin{array}{c}\mathcal{N}(x)=\emptyset \text { or } \exists N(x): \\
N \in \mathcal{N}(x) \\
\Longleftrightarrow N(x) \subseteq N\end{array}$ \\
\hline
\end{tabular}

Almost all approaches to extend the framework of topology at least assume that the closure functions are isotonic, or, equivalently, that the neighborhoods of a point form a "stack", see e.g. $[8,14,31,35,36]$. The importance of isotony is emphasized by several equivalent conditions, see e.g. [36, Lem.10]:

(K1) $A \subseteq B$ implies $\mathrm{cl}(A) \subseteq \mathrm{cl}(B)$ for all $A, B \in \mathcal{P}(X)$.

$\left(\mathrm{K} 1^{\prime}\right) \mathrm{cl}(A) \cup \mathrm{cl}(B) \subseteq \mathrm{cl}(A \cup B)$ for all $A, B \in \mathcal{P}(X)$.

$\left(\mathrm{K} 1^{\prime \prime}\right) \operatorname{cl}(A \cap B) \subseteq \mathrm{cl}(A) \cap \mathrm{cl}(B)$

A (not necessarily non-empty) collection $\mathcal{F} \subseteq \mathcal{P}(X)$ is a stack if $F \in \mathcal{F}$ and $F \subseteq G$ implies $G \in \mathcal{F}$. Let us write $\mathfrak{S}(X)$ for the set of all stacks. It is important to distinguish the empty set $\emptyset \in \mathcal{P}(X)$ and the empty stack $\varnothing \subseteq$ $\mathcal{P}(X)$. There is a condition equivalent to (K1) in terms of the neighborhood function: The closure functions $\mathrm{cl}$ is isotonic if and only if $\mathcal{N}(x)$ is a stack for all $x \in X$.

Kuratowski's axioms for the closure function of a topological [51] may be seen as specializations of the very general closure functions that we have considered so far:

Let $(X, \mathrm{cl})$ be a generalized closure space and consider the following properties 
Table 2

Axioms for various types of closure functions.

Defining axioms are marked by $\bullet$, further properties that implied are marked by $\circ$.

\begin{tabular}{|c|c|c|c|c|c|c|c|}
\hline Axiom & $\frac{\Theta}{\underbrace{\theta}_{U}}$ & 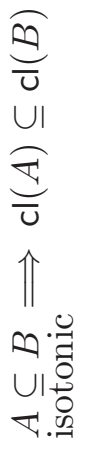 & 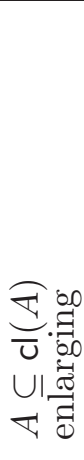 & 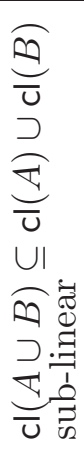 & 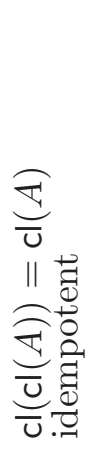 & 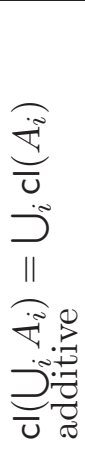 & Ref. \\
\hline Extended Topology & $\bullet$ & $\bullet$ & & & & & {$[36]$} \\
\hline Brissaud & $\bullet$ & & $\bullet$ & & & & [8] \\
\hline Neighborhood space & $\bullet$ & $\bullet$ & • & & & & {$[38]$} \\
\hline Closure space & $(\bullet)$ & $\bullet$ & $\bullet$ & & $\bullet$ & & {$[62]$} \\
\hline Smyth space & $\bullet$ & $\bullet$ & & $\bullet$ & & & {$[61]$} \\
\hline Binary relation & $\bullet$ & ○ & & $\circ$ & & - & {$[9,52]$} \\
\hline Pretopology & $\bullet$ & $\bullet$ & $\bullet$ & $\bullet$ & & & {$[10]$} \\
\hline Topology & $\bullet$ & $\bullet$ & $\bullet$ & $\bullet$ & $\bullet$ & & \\
\hline Alexandroff space & $\bullet$ & ० & $\bullet$ & $\circ$ & & - & \\
\hline Alexandroff topology & $\bullet$ & ० & $\bullet$ & $\circ$ & $\bullet$ & $\bullet$ & {$[1]$} \\
\hline
\end{tabular}

of the closure function for all $A, B \in \mathcal{P}(X)$.

(K0) $\mathrm{cl}(\emptyset)=\emptyset$

(K1) $A \subseteq B$ implies $\mathrm{cl}(A) \subseteq \mathrm{cl}(B)$ (isotonic).

(K2) $A \subseteq \mathrm{cl}(A)$ (expanding).

(K3) $\mathrm{cl}(A \cup B) \subseteq \mathrm{cl}(A) \cup \mathrm{cl}(B)$ (sub-additive).

(K4) $\mathrm{cl}(\mathrm{cl}(A))=\mathrm{cl}(A)$ (idempotent).

(K5) $\bigcup_{i \in I} \mathrm{cl}\left(A_{i}\right)=\mathrm{cl}\left(\bigcup_{i \in I} A_{i}\right)$ (additive).

The conditions in each row of table 1 are equivalent.

Different combinations of these axioms define topological structures that have been studied to various degrees in the literature; table 2 summarizes the best known ones.

Dikranjan et al. [15] show that the class of generalized closure space $(X, \mathrm{cl})$ 
satisfying (K0), (K1), (K2), and (K4) form a topological category. It is well known that the Cech closure spaces, which satisfy (K0), (K1), (K2), and (K3), are identical to the pretopological spaces which also form a topological category, see e.g. [56].

An extensive textbook by Eduard Čech [10] demonstrates that much of the classical theory of point set topology remains intact in pretopological spaces, i.e., when the assumption that the closure is idempotent is dropped. As a consequence, the notions of open and closed sets play little actual role in this theory - quite in contrast to usual way this mathematical theory is taught.

Further generalization have received much less attention. In neighborhood spaces, for example, much of the hierarchy of separation axioms is still intact [65], while other important results, such as Urysohn's lemma, fail [69]. As we shall see in the following section, one has to be even more careful in the case of isotonic spaces.

\section{Connectedness}

Topological connectedness is closely related to separation. Two sets $A, B \in$ $\mathcal{P}(X)$ are semi-separated if there are neighborhoods $N^{\prime} \in \mathcal{N}(A)$ and $N^{\prime \prime} \in$ $\mathcal{N}(B)$ such that $A \cap N^{\prime \prime}=N^{\prime} \cap B=\emptyset$; they are separated if if there are neighborhoods $N^{\prime} \in \mathcal{N}(A)$ and $N^{\prime \prime} \in \mathcal{N}(B)$ such that $N^{\prime} \cap N^{\prime \prime}=\emptyset$.

A set $Z \in \mathcal{P}(X)$ is connected in a space $(X, \mathrm{cl})$ if it is not a disjoint union of a nontrivial semi-separated pairs of sets $A, Z \backslash A, A \neq \emptyset, Z$.

For proofs of the statements in this paragraph we refer to the supplementary material of [64]. If $(X, \mathrm{cl})$ is isotone then $A$ and $B$ are semi-separated if and only if $\mathrm{cl}(A) \cap B=A \cap \mathrm{cl}(B)=\emptyset$. Connectedness in isotonic spaces can thus be characterized by the Hausdorff-Lennes condition: A set $Z \in \mathcal{P}(X)$ is connected in an isotonic space $(X, \mathrm{cl})$ if and only if for each proper subset $A \subseteq Z$ holds

$$
[\mathrm{cl}(A) \cap(Z \backslash A)] \cup[\mathrm{cl}(Z \backslash A) \cap A] \neq \emptyset
$$

The collection of connected sets satisfies the following three properties in isotonic spaces [34]:

(c1) If $Z$ consists of a single point, then $Z$ is connected.

(c2) If $Y$ and $Z$ are connected and $Y \cap Z \neq \emptyset$ then $Y \cup Z$ is connected

(c3) If $Z$ is connected, then $\mathrm{cl}(Z)$ is also connected.

There have been several attempts to use connectedness as the primitive notion in topological theory $[37,39,74]$. 
This topological notion of connectedness nicely coincides with the notion of connectedness in directed graphs: In this case $\mathrm{cl}(A)$ is the set of out-neighbors of the vertices $x \in A$ plus $A$ itself. Thus $A$ is connected unless there is a nontrivial bi-partition $A^{\prime}, A^{\prime \prime}$ of $A$ without an arrow pointing from $A^{\prime}$ to $A^{\prime \prime}$ or vice versa. It has severe shortcomings, however, in more general neighborhood spaces. Intuitively, one would like to consider subspaces of $(X, p)$ that are spanned by a single reaction as connected. This is in general not the case, however. Consider $X=\{u, v, x, y\}$ with a single reaction $\rho: u+v \rightarrow x+y$. One easily checks that $A=\{u, x\}$ and $\bar{A}=\{v, y\}$ form a semi-separation, hence $X$ is not connected, see also Fig. 8. Topological connectedness is thus too restrictive in the context of chemical reaction networks.

Another natural notion of connectedness derives from the hypergraph representation $[68,76]$ of $(X, \mathfrak{R})$ : We might say that a set is "graphically connected" if it is connected in the "substrate graph" of $(X, \mathfrak{R})[72]$, which is obtained by connecting two molecules $x$ and $y$ whenever they are involved in the same reaction $\rho$, i.e., $\{x, y\}$ is an edge if and only if there is a reaction $\rho$ such that $\{x, y\} \subseteq \operatorname{dom} \rho \cup \operatorname{img} \rho$. This notion of connectedness, however, does not really seem very useful, since it is easy to construct quite large graphically connected sets on which not a single reaction is possible. To see this, we consider the following construction. We start from

$$
B_{x}=\bigcup_{\rho: x \in \operatorname{dom} \rho}(\operatorname{dom} \rho \cup \operatorname{img} \rho)
$$

and remove molecules $y \neq x$ until $\operatorname{dom} \rho \nsubseteq B^{\prime}$ for any $\rho$. By construction, each $y \in B^{\prime}$ is connected to $x$ in the substrate graph, thus $B^{\prime}$ is graphicall connected. In general, $B^{\prime}$ can be a very large set.

As a middle ground we propose to consider a more restrictive notions of separation between sets. This concept then implies a less restrictive notion of connectedness.

We say that $A, B \in \mathcal{P}(X)$ are productively separated if for all $Z \subseteq A \cup B$ holds (1) $\mathrm{cl}(Z \cap A) \cap B=\emptyset$ and $\mathrm{cl}(Z \cap B) \cap A=\emptyset$

(2) $\mathrm{cl}(Z)=\mathrm{cl}(Z \cap A) \cup \mathrm{cl}(Z \cap B)$.

If $(X, \mathrm{cl})$ is an isotonic space, then $A$ and $B$ are semi-separated if condition (1) holds for all $Z \subseteq A \cup B$. (To see this observe that isotony implies that

(1) is true for all $Z \subseteq A \cup B$ whenever it is true for $Z=A \cup B$.) Condition (2) becomes trivial in additive spaces. In non-additive spaces, however, (2) encapsulates an important property: From two productively separated sets we cannot produce anything that we cannot obtain from the individual sets. In terms of our chemical networks, there are no reaction products that we can obtain only by mixing $A$ and $B$.

Lemma 8 Suppose $A$ and $B$ are productively separated in an isotonic space 

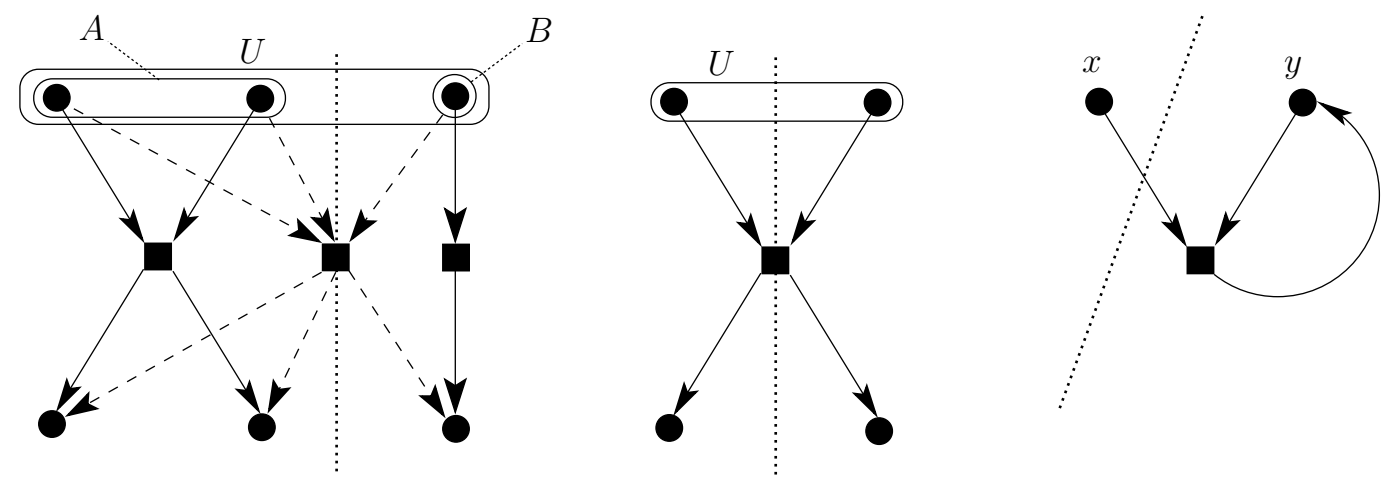

Fig. 8. Illustration of the concepts seperated, semiseperated and productivly connected.(left chart) The reaction network is seperated although from a graph theoretical view point the network is connected. This classification makes sense since in a chemical sense nothing new is gained by the super reaction (dashed arrows) which holds the network graph theoretically together.(chart middle) The reaction network is semi-seperated. (right chart) The reaction network is productively connected with respect to $p^{\prime}$ since somthing new is produced from the union of the subsets $\left(p^{\prime}(\{x\})=p^{\prime}(\{y\})=p^{\prime}(\{x\}) \cup p^{\prime}(\{y\})=\emptyset\right.$ and $\left.p^{\prime}(\{x\} \cup\{y\})=\{y\}\right)$. With respect to $p$ the reaction network is not productively connected because nothing new can be produced that was not already part of one of the two subsets $(p(\{x\})=\{x\}, p(\{y\}=\{y\}$ and $p(\{x\}) \cup p(\{y\}=p(\{x\} \cup\{y\})=\{x, y\})$.

$(X, \mathrm{cl})$. Let $A^{\prime} \subseteq A$ and $B^{\prime} \subseteq B$ be non-empty. Then $A^{\prime}$ and $B^{\prime}$ are also productively separated.

Proof: Follows directly from the definition (choosing $Z=A^{\prime} \cup B^{\prime}$ ).

It is now natural to call a set $Z$ productively connected if it cannot be decomposed into two non-empty subsets $Z^{\prime}$ and $Z^{\prime \prime}=Z \backslash Z^{\prime}$ with $Z^{\prime} \cap Z^{\prime \prime}=\emptyset$ that are productively separated. In general, if $Z$ is connected, then it is also productively connected. In pretopological spaces (and in particular in digraphs), semi-separation and productive separation coincide, hence $Z$ is productively connected if and only if it is connected in this case.

The following lemma shows that productive connectedness matches much closer our intuition of a "connected" reaction network. Indeed, most individual reactions correspond to connected sets:

Lemma 9 Consider a chemical universe $(X,\{\rho\})$ with $\operatorname{dom} \rho \cup \operatorname{img} \rho=X$ and $\operatorname{dom} \rho \neq X$. Then $(X,\{\rho\})$ is productively connected w.r.t. the closure function $p$.

Proof: Consider a proper split $\{A, \bar{A}\}$ of $X$, i.e., $A \cup \bar{A}=X, A \cap \bar{A}=\emptyset$, and both $A$ and $\bar{A}$ are non-empty. We can distinguish two major cases: (i) $\operatorname{dom} \rho \subseteq A$ and (ii) $\operatorname{img} \rho \backslash A$ and $A \backslash \operatorname{img} \rho$ are both non-empty. The third case, 
$A \cap \operatorname{img} \rho=\emptyset$, reduces to case (i) by exchanging $A$ and $\bar{A}$.

Case (i): we have $X=p(\operatorname{dom} \rho) \subseteq p(A)$, i.e., $p(A) \cap \bar{A} \neq \emptyset$, and hence the split $\{A, \bar{A}\}$ is not a semi-separation of $X$.

Case (ii): we have $p(A)=A$ and $p(\bar{A})=\bar{A}$, i.e., the split $\{A, \bar{A}\}$ is a semiseparation. There is, however, $Z=\operatorname{dom} \rho$ such that $p(Z \cap A)=Z \cap A, p(Z \cap$ $\bar{A})=Z \cap \bar{A}$, and hence $p(Z \cap A) \cup p(Z \cap \bar{A})=Z$. On the other hand, $p(Z)=X$. By assumption, $Z \neq X$. Thus the split $\{A, \bar{A}\}$ is not productive semi-separation of $X$.

It follows that $(X,\{\rho\})$ does not admit a productive semi-separation w.r.t. $p$, hence it is productively connected w.r.t. to $p$.

Note that $(X,\{\rho\})$ is in general not connected w.r.t $p$ if $\operatorname{img} \rho \subseteq \operatorname{dom} \rho=X$, Fig. 8.

It seems natural to call a reaction "topologically elementary" if (i) img $\rho \nsubseteq$ $\operatorname{img} \rho$ and (ii) there is no other reaction $\rho^{\prime}$ with $\operatorname{dom} \rho^{\prime} \cup \operatorname{img} \rho^{\prime} \subseteq \operatorname{dom} \rho \cup \operatorname{img} \rho$ with the exception of the reverse reaction $\rho^{\prime}=\bar{\rho}$. The following theorem shows that productively connected sets can be built up from overlapping "topologically elementary" reactions. It will be an interesting question to characterize the connected sets that are spanned by individual topologically nonelementary reactions.

Theorem 5 In neighborhood spaces, productive connectedness has the following properties:

(c1) If $Z$ consists of a single point, then $Z$ is productively connected.

(c2) If $Y$ and $Z$ are productively connected and $Y \cap Z \neq \emptyset$ then $Y \cup Z$ is connected

(c3) If $Z$ is productively connected, then $\mathrm{cl}(Z)$ is also productively connected.

(c4) Let $\left\{Z_{i} \mid i \in I\right\}$ be an arbitrary collection of connected sets such that $\bigcap_{i \in I} Z_{i} \neq$ $\emptyset$. Then $W:=\bigcup_{i \in I} Z_{i}$ is connected.

Proof: Property (c1) is trivial since there is no non-trivial partition.

Suppose that $Y \cup Z$ can be decomposed into two non-empty productively separated sets $P$ and $Q$. By Lemma $8 P \cap Y$ and $Q \cap Y$ are also productively separated unless one of the intersections is empty. Since $Y$ is assumed to be productively connected, we have to assume that one of the intersections is empty. The same argument can be made for $Z$. Thus the only possibility is $Y \subseteq P$ and $Z \subseteq Q$ (or vice versa). On the other hand, we have $Y \cap Z=\emptyset$. It follows that $Y \cup Z$ must be productively connected.

A similar argument shows that $\mathrm{cl}(Y)$ is connected. Again, suppose $C A=P \cup Q$ for two productively separated sets $P$ and $Q$. In a neighborhood space, $Y \subseteq$ $\mathrm{cl}(Y)$. Thus $P \cap Y$ and $Q \cap Y$ are semiseparated unless one of them is empty. It follows that either $Y \subseteq P$ or $Y \subseteq Q$. But then either $\mathrm{cl}(P)$ or $\operatorname{cl}(Q)$ contains $\mathrm{cl}(Y)$ by isotony, i.e., either $Q$ or $P$ is empty, a contradiction. Hence $\mathrm{cl}(Y)$ is productively connected. 
Suppose $W$ is not connected. Then there is a productive separation $W=$ $W^{\prime} \dot{\cup} W^{\prime \prime}$ with nonempty sets $W^{\prime}$ and $W^{\prime \prime}$. Observe that in this case $Z_{i}^{\prime}=$ $Z_{i} \cap W^{\prime}$ and $Z_{i}^{\prime}=Z_{i} \cap W^{\prime}$ for a productive separation of $Z_{i}$ unless either $Z_{i}^{\prime}$ or $Z_{i}^{\prime \prime}$ is empty. By construction, there is a point $z \in Z_{i}$ for all $i \in I$. W.l.o.g. we may assume $z \in W^{\prime}$. This implies $Z_{i}^{\prime \prime}=\emptyset$ and hence $Z_{i} \subseteq W^{\prime}$ for all $i$, and hence $W^{\prime \prime}=\emptyset$, contradicting the assumption that $W$ admits a productive separation. Therefore, $W$ is connected.

It follows immediately that for each set $A$ and each point $x \in A$ the connected component $A[x]$ of $A$ that contains $x$ is well defined:

$$
A[x]=\bigcup\left\{A^{\prime} \subset A \mid x \in A^{\prime} \text { and } A^{\prime} \text { is connected }\right\}
$$

We see that productive connectedness provides an generalization of the usual notion of connectedness in neighborhood spaces that has the same convenient mathematical properties and a more intuitive interpretation. Since the two concepts coincide for the much better studies pretopological and topological spaces, one might argue that productive connectedness is the most natural notion of connectedness in neighborhood spaces.

\section{Conclusion and Outlook}

We have shown here how the stoichiometric picture of a chemical reaction network can be interpreted within the framework of point-set topology. Specifically, we consider a set of molecular species a (generalized) topological space, whose structure is determined by mutual accessibility via chemical reactions. The translation of concepts from chemical reaction network theory to this much more abstract mathematical framework gives access to a rich "language" and a collection of concepts - including connectedness (a focus in this study), compactness, convergence, regularity, and uniformity - that have been developed and explored by mathematicians for more than a century. We have demonstrated here in some detail how these topological notions can be translated back to the concrete case of chemical reaction networks. As our understanding of structural properties of large-scale chemical networks is still in its infancy, it remains an interesting research agenda to explore the chemical meaning of these abstract concepts in chemical reaction network theory. The same basic mathematical theory describes e.g. search spaces in molecular evolution as well as in combinatorial optimization settings that use complex search operators such as cross-over [67].

The basic "building blocks" of the topological approach are set-valued setfunctions that describe which molecules can be generated from a given seed 
set of compounds. These functions implictly arrange the "chemical universe" is a way that places molecules close to each other if they can interconverted by applying few elementary reactions. Chemical organization theory [16] naturally can be re-formulated in the topological language, with organization taking on the role of appropriately defined closed sets.

The reformulation of chemical organization theory in topological terms and the theorems derived here have also practical implications. The definition of self-maintenance in terms of a set-valued set function highlights algebraic properties of this functions that in turn suggest novel avenues for algorithmic improvements. For instance, our results show that self-maintenance can be verified by checking check each molecule separately whether is is maintainable, instead of trying to find a flux vector that maintains all molecules at once. Even if this does not lead to faster algorithms, we might use the concepts of maintainable (and stationarily produced) as a measure of the degree of maintainability of a set of molecules. For instance, the relative size of the set of stationarily produced molecules can be taken as a structural measure of robustness of an organization, because stationarily produced molecules can be regenerated when destroyed. Furthermore we can take the new concepts to explain why a set is not self-maintaining, by identifying those molecules that are not maintainable.

The topological notion of neighborhood, separateness, and productively connectedness are rather abstract and yet difficult to comprehend intuitively from a biochemists point of view. Nevertheless we have shown that they lead to rather natural or at least interesting characterizations of the structure of a network. A challenging task will be to find their right interpretations and application range, which will be a perquisite to make the theory accessible through software tools.

Network comparison and network alignment are interesting potential application area of our approach. Forst et al. [27] have shown that the extension of set algebra to chemical reaction networks opens the route for the identification of distinct metabolic features in two sets of organisms. Yet it is unclear if this has also interesting consequences in the topological framework.

The topological interpretation of chemical reaction networks and their organizations emphasizes the distinction between structural and dynamical properties since dynamics has to be understood as a process living on the underlying topological structures. Exploring the relation of structural features of the network to dynamical properties - as it has been done for chemical organizations ([16], Theorem 1) - has been identified an important element of future research. For instance, Feinberg and collaborators [12] recently characterized those mass-action networks that can support bi-stable behavior on the basis of structural features of the "species reaction graph". It will be interesting to 
see if their results have a natural topological interpretation as well.

As sketched above, there are various open problems, which cumulate into the fundamental question how topological properties are related to dynamical behavior, or vice versa, how observed dynamics can be explained in terms of local topological properties of the network.

\section{Acknowledgments}

This work owes its existence to a number of form-free environments far away from office desks, computers, telephones, and emails: the beaches of Brighton (England), Ulcin (Montenegro), Oahu, and Hawai'i, and the winter landscape at Lake Bled in Slovenia. Financial support by the DFG Gradiertenkolleg Wissenrepräsentation to GB is gratefully acknowledged.

\section{References}

[1] P. Alexandroff. Diskrete Räume. Math. Sb. (N.S.), 2:501-518, 1937.

[2] R. J. Bagley and J. D. Farmer. Spontaneous emergence of a metabolism. In C. G. Langton, C. Taylor, J. D. Farmer, and S. Rasmussen, editors, Artificial Life II, Santa Fe Institute Studies in the Sciences of Complexity, pages 93-141, Redwood City, CA, 1992. Addison-Wesley.

[3] W. Banzhaf, P. Dittrich, and B. Eller. Self-organization in a system of binary strings with spatial interactions. Physica D, 125:85-104, 1999.

[4] G. Benkö. Uncovering the Structure of an Artificial Chemistry Universe. PhD thesis, Faculty for Mathematics and Computer Science, University of Leipzig, 2006 .

[5] G. Benkö, C. Flamm, and P. F. Stadler. Generic properties of chemical networks: Artificial chemistry based on graph rewriting. In W. Banzhaf, T. Christaller, P. Dittrich, J. T. Kim, and J. Ziegler, editors, Advances in Artificial Life, volume 2801 of Lecture Notes in Computer Science, pages 10-20, Heidelberg, Germany, 2003. Springer-Verlag. Proceedings of the 7th European Conference of Artifical Life, ECAL 2003, Dortmund, Germany, September 1417, 2003, Proceedings.

[6] G. Benkö, C. Flamm, and P. F. Stadler. A graph-based toy model of chemistry. J. Chem. Inf. Comput. Sci., 43:1085-1093, 2003.

[7] G. Berry and G. Boudol. The chemical abstract machine. Theor. Comp. Sci., 96:217-248, 1992. 
[8] M. M. Brissaud. Les espaces prétopologiques. C. R. Acad. Sc. Paris Ser. A, 280:705-708, 1975.

[9] C. Calude and V. E. Căzănescu. On topologies generated by Mosil resemblance relations. Discr. Math., 25:109-115, 1979.

[10] E. Čech. Topological Spaces. Wiley, London, 1966.

[11] B. L. Clarke. Stoichiometric network analysis. Cell Biophys., 12:237-253, 1988.

[12] G. Craciun, Y. Tang, and M. Feinberg. Understanding bistability in complex enzyme-driven reaction networks. Proc. Natl. Acad. Sci. USA, 103:8697-8702, 2006 .

[13] J. Cupal, S. Kopp, and P. F. Stadler. RNA shape space topology. Artificial Life, 6:3-23, 2000.

[14] M. M. Day. Convergence, closure, and neighborhoods. Duke Math. J., 11:181199, 1944.

[15] D. Dikranjan, E. Guili, and A. Tozzi. Topological categories and closure operators. Quest. Math., 11:323-337, 1988.

[16] P. Dittrich and P. Speroni di Fenizio. Chemical organization theory: towards a theory of constructive dynamical systems. Bull. Math. Biol., 2006. arXiv.org q-bio.MN/0501016.

[17] P. Dittrich, J. Ziegler, and W. Banzhaf. Artificial chemistries - a review. Artificial Life, 7:225-275, 2001.

[18] J. Dugundji and I. Ugi. Theory of the be- and r-matrices. Top. Curr. Chem., 39:19-29, 1973.

[19] M. Feinberg. Complex balancing in general kinetic systems. Arch. Rational Mech. Anal., 49:187-194, 1972.

[20] M. Feinberg and F. J. M. Horn. Dynamics of open chemical systems and the algebraic structure of the underlying reaction network. Chem. Eng. Sci., 29(3):775-787, 1973.

[21] D. A. Fell. Understanding the Control of Metabolism. Portland Press, London, 1997.

[22] W. Fontana. Algorithmic chemistry. In C. G. Langton, C. Taylor, J. D. Farmer, and S. Rasmussen, editors, Artificial Life II, pages 159-210, Redwood City, CA, 1992. Addison-Wesley.

[23] W. Fontana and L. W. Buss. "the arrival of the fittest":towards a theory of biological organisation. Bull. Math. Biol., 56(1):1-64, 1994.

[24] W. Fontana and L. W. Buss. What would be conserved if 'the tape were played twice'? Proc. Natl. Acad. Sci. USA, 91:757-761, 1994.

[25] W. Fontana and P. Schuster. Continuity in Evolution: On the Nature of Transitions. Science, 280:1451-1455, 1998. 
[26] W. Fontana and P. Schuster. Shaping Space: The Possible and the Attainable in RNA Genotype-Phenotype Mapping. J. Theor. Biol., 194:491-515, 1998.

[27] C. V. Forst, C. Flamm, I. L. Hofacker, and P. F. Stadler. Algebraic comparison of metabolic networks, phylogenetic inference, and metabolic innovation. $B M C$ Bioinformatics, 7:67, 2006.

[28] J. Gagneur and S. Klamt. Computation of elementary modes: a unifying framework and the new binary approach. BMC Bioinformatics, 5:175 [epub], 2004 .

[29] G. C. Gastl and P. C. Hammer. Extended topology. Neighborhoods and convergents. In N.N., editor, Proceedings of the Colloquium on Convexity 1965, pages 104-116, Copenhagen, DK, 1967. Københavns Univ. Matematiske Inst.

[30] P. M. Gleiss, P. F. Stadler, A. Wagner, and D. A. Fell. Relevant cycles in chemical reaction network. Adv. Complex Syst., 4:207-226, 2001.

[31] S. Gniłka. On extended topologies. I: Closure operators. Ann. Soc. Math. Pol., Ser. I, Commentat. Math., 34:81-94, 1994.

[32] S. Gniłka. On continuity in extended topologies. Ann. Soc. Math. Pol., Ser. I, Commentat. Math., 37:99-108, 1997.

[33] J. M. Guberman. Mass action networks and the deficiency zero theorem. Master's thesis, Department of Mathematics, Harvard University, 2003.

[34] E. D. Habil and K. A. Elzenati. Connectedness in isotonic spaces. Turk. J. Math, 2006. in press.

[35] P. C. Hammer. General topology, symmetry, and convexity. Trans. Wisconsin Acad. Sci., Arts, Letters, 44:221-255, 1955.

[36] P. C. Hammer. Extended topology: Set-valued set functions. Nieuw Arch. Wisk. III, 10:55-77, 1962.

[37] P. C. Hammer. Extended topology: Connected sets and Wallace separations. Portug. Math., 22:77-93, 1963.

[38] P. C. Hammer. Extended topology: Continuity I. Portug. Math., 25:77-93, 1964.

[39] J. M. Harris. Continuity and separation for point-wise symmetric isotonic closure functions. Technical report, 2005. arXiv:math.GN/0507230.

[40] R. Heinrich and T. A. Rapoport. A linear steady-state treatment of enzymatic chains. General properties, control and effector strengths. Eur. J. Biochem., 42:97-105, 1974.

[41] R. Heinrich and S. Schuster. The Regulation of Cellular Systems. Chapman and Hall, 1996.

[42] J. Hofbauer and K. Sigmund. Dynamical Systems and the Theory of Evolution. Cambridge University Press, Cambridge U.K., 1988. 
[43] R. Höllering, J. Gasteiger, L. Steinhauer, K. Schulz, and A. Herwig. The simulation of organic reactions: From the degradation of chemicals to combinatorial synthesis. J. Chem. Inf. Comput. Sci., 40:482-494, 2000.

[44] W. Hordijk and J. F. Fontanari. Catalytic reaction sets, decay, and the preservation of information. In Proceedings of the IEEE International Conference on Integration of Knowledge Intensive Multi-Agent Systems, pages 133-138, 2003.

[45] W. Hordijk and M. Steel. Detecting autocatalytic, self-sustaining sets in chemical reaction systems. J. Theor. Biol., 227:451-461, 2004.

[46] F. Horn. Necessary and sufficient conditions for complex balancing in chemical kinetics. Arch. Rational Mech. Anal., 49:172-186, 1972.

[47] F. Horn and R. Jackson. General mass action kinetics. Arch. Rational Mech. Anal., B47:81-116, 1972.

[48] H. Kacser and J. A. Burns. The control of flux. Symp. Soc. Exp. Biol., 32:65$104,1973$.

[49] H. Kacser, J. A. Burns, and D. A. Fell. The control of flux: 21 years on. Biochem. Soc. Trans., 23:341-366, 1995.

[50] S. A. Kauffman. The Origin of Order. Oxford University Press, New York, Oxford, 1993.

[51] C. Kuratowski. Sur la notion de limite topologique d'ensembles. Ann. Soc. Polon. Math., 21:219-225, 1949.

[52] M. Malitza. Topology, binary relations, and internal operations. Rev. Roumaine Math. Pures Appl., 4:515-519, 1975.

[53] J. S. McCaskill and U. Niemann. Graph replacement chemistry for DNA processing. In A. Condon and G. Rozenberg, editors, DNA Computing, volume 2054 of Lecture Notes in Computer Science, pages 103-116. Springer, Berlin, D, 2000.

[54] J. S. Olivera, C. G. Bailey, J. B. Jones-Olivera, and D. A. Dixon. An algebraic-combinatorial model for the identification and mapping of biochemical pathways. Bull. Math. Biol., 63:1163-1196, 2001.

[55] C. A. Petri. Kommunikation mit Automaten. PhD thesis, University of Bonn, Bonn, 1962.

[56] G. Preuß. Convenient topology. Math. Japonica, 47:171-183, 1998.

[57] C. H. Schilling, D. Letscher, and B. Ø. Palsson. Theory for the systematic definition of metabolic pathways and their use in interpreting metabolic function from a pathway-oriented perspective. J. Theor. Biol., 203:229-248, 2000.

[58] C. H. Schilling and B. Ø. Palsson. The underlying pathway structure of biochemical reaction networks. Proc. Natl. Acad. Sci. USA, 95:4193-4198, 1998. 
[59] S. Schuster, D. A. Fell, and T. Dandekar. A general definition of metabolic pathways useful for systematic organization and analysis of complex metabolic networks. Nature Biotechnol., 18:326-332, 2000.

[60] P. H. Sellers. An Introduction to a Mathematical Theory of Chemical Reaction Networks I\&II. Arch. Rat. Mech. Anal., 44:23-40, 376-386, 1971/1972.

[61] M. B. Smyth. Semi-metric, closure spaces and digital topology. Theor. Computer Sci., 151:257-276, 1995.

[62] W. P. Soltan. An Introduction in Axiomatic Theory of Convexity. Shtiintsa, Kishinev, 1984. Russian.

[63] P. Speroni di Fenizio. A less abstract artificial chemistry. In M. Bedau, J. McCaskill, N. Packard, and S. Rasmussen, editors, Artificial Life VII, pages 49-53, Cambridge, MA, 2000. MIT Press.

[64] B. M. R. Stadler and P. F. Stadler. Generalized topological spaces in evolutionary theory and combinatorial chemistry. J. Chem. Inf. Comput. Sci., 42:577-585, 2002. Proceedings MCC 2001, Dubrovnik.

[65] B. M. R. Stadler and P. F. Stadler. Higher separation axioms in generalized closure spaces. Commentationes Math. Warszawa, Ser. I, 43:257-273, 2003.

[66] B. M. R. Stadler, P. F. Stadler, M. Shpak, and G. P. Wagner. Recombination spaces, metrics, and pretopologies. Z. Phys. Chem., 216:217-234, 2002.

[67] B. M. R. Stadler, P. F. Stadler, G. P. Wagner, and W. Fontana. The topology of the possible: Formal spaces underlying patterns of evolutionary change. $J$. Theor. Biol., 213:241-274, 2001.

[68] O. N. Temkin, A. V. Zeigarnik, and D. G. Bonchev. Chemical Reaction Networks: A Graph-Theoretical Approach. CRC Press, Boca Raton, FL, 1996.

[69] D. Thampuran. Normal neighborhood spaces. Rend. Sem. Mat. Univ. Padova, 45:95-97, 1971.

[70] M. Thürk. Ein Modell zur Selbstorganisation von Automatenalgorithmen zum Studium molekularer Evolution. PhD thesis, Universität Jena, Germany, 1993. PhD Thesis.

[71] I. Ugi, N. Stein, M. Knauer, B. Gruber, K. Bley, and R. Weidinger. New elements in the representation of the logical structure of chemistry by qualitative mathematical models and corresponding data structures. Top. Curr. Chem., 166:199-233, 1993.

[72] A. Wagner and D. A. Fell. The small world inside large metabolic networks. Proc. Royal Soc. London B, 268:1803-1810, 2001.

[73] G. Wagner and P. F. Stadler. Quasi-independence, homology and the unity of type: A topological theory of characters. J. Theor. Biol., 220:505-527, 2003.

[74] A. D. Wallace. Separation spaces. Ann. Math., pages 687-697, 1941. 
[75] Y. L. Yung and W. B. DeMore. Photochemistry of Planetary Athmospheres. Oxford University Press, New York, 1999.

[76] A. V. Zeigarnik. On hypercycles and hypercircuits in hypergraphs. In P. Hansen, P. W. Fowler, and M. Zheng, editors, Discrete Mathematical Chemistry, volume 51 of DIMACS series in discrete mathematics and theoretical computer science, pages 377-383. American Mathematical Society, Providence, RI, 2000. 